\title{
The quantification and monitoring of intraoperative nociception levels in thoracic surgery: a review
}

\author{
Ismael Ghanty, Stefan Schraag \\ Department of Perioperative Medicine, Golden Jubilee National Hospital, Clydebank, UK \\ Contributions: (I) Conception and design: S Schraag; (II) Administrative support: None; (III) Provision of study materials or patients: None; (IV) \\ Collection and assembly of data: None; (V) Data analysis and interpretation: None; (VI) Manuscript writing: All authors; (VII) Final approval of \\ manuscript: All authors. \\ Correspondence to: Stefan Schraag, MD, PhD, FRCA, FFICM. Professor of Anaesthesia, Consultant Cardiothoracic Anaesthetist, Department of \\ Perioperative Medicine, Golden Jubilee National Hospital, Clydebank G81 4NE, UK. Email: stefanschraag@icloud.com.
}

\begin{abstract}
Nociception is the unconscious perception of a stimulus applied by trauma or surgery and expressed through a response of the autonomous nervous system. Local anaesthetics (LAs), opioids and other modulating agents such as ketamine are usually utilised to blunt nociception as a component during general anaesthesia (GA) and surgery. The effectiveness of these measures, however, are still difficult to quantify and monitoring of anti-nociception has been confined to assess variation of heart rate (HR) or blood pressure (BP). Recently, various monitoring concepts have been introduced to quantify nociception more systematically and on the other hand guide anti-nociceptive interventions more appropriately. This review describes the various technologies, their performance in clinical studies and provides a critical appraisal with particular application to thoracic anaesthesia and surgery and their relevance in the context of chronic pain after surgery.
\end{abstract}

Keywords: Thoracic anaesthesia; robot-assisted thoracic surgery; nociception; monitoring

Submitted Mar 29, 2019. Accepted for publication Aug 13, 2019.

doi: $10.21037 /$ jtd.2019.08.62

View this article at: http://dx.doi.org/10.21037/jtd.2019.08.62

\section{Introduction}

General anaesthesia (GA) is a pharmacologically-induced reversible state of unconsciousness, amnesia, analgesia (or antinociception), and immobility (1).

The level of the hypnotic component of anaesthesia can be routinely monitored using indices derived from processed electroencephalogram (EEG) signals. These include the Bispectral Index $\left(\mathrm{BIS}^{\circledR}\right)$ (2), or the Spectral Entropy (including State entropy and Response Entropy) (3) as well as visual assessment of the power spectrum. It should be noted that there are limitations to the use of these indices in assessing all aspects of anaesthesia, which are discussed elsewhere (4-6).

Likewise, neuromuscular blockade can be assessed with quantitative peripheral nerve stimulators. These can confirm adequate muscle relaxation before endotracheal intubation, and test for residual neuromuscular blockade postoperatively, with an objective measurement [train of four (TOF) $>0.9$ ] determining appropriate timing of tracheal extubation without residual paralysis.

Conversely, objectively assessing nociception induced by (and during) surgery has been more challenging to achieve. Here the distinction between pain and nociception needs to be made. Pain is the conscious perception of (potentially) noxious stimuli (7). Therefore, during the unconscious state of anaesthesia, we refer to nociception, that is, the neural conduction and processing of noxious stimuli in the central nervous system. During surgery, there is ideally a 'balance' between the degree of nociceptive stimuli and the antinociceptive component of GA.

Over the past two decades, a number of non-invasive techniques have been developed to detect and 'quantify' the intraoperative nociception-antinociception (NAN) balance, with several commercial monitors now available. However, they are not yet routinely utilised, especially compared to 
the EEG-based indices. This review will look at some of the existing techniques, with a focus on the studies investigating their clinical use in thoracic anaesthesia and surgery.

\section{Chronic pain after thoracic surgery}

Although chronic pain is a recognised problem after different surgical procedures, it is particularly common after thoracic surgery. A meta-analysis performed in 2014 estimated the incidence of chronic pain at 3 and 6 months after thoracotomy as $57 \%$ and $47 \%$ respectively (8). These rates have been grossly stable since the 1990s, despite improvements in perioperative care.

Over the past three decades, there has been a shift towards minimally invasive approaches to thoracic surgery, such as VATS, and more recently, RATS with the da Vinci surgical system (Intuitive Surgery, Sunnyvale, CA, USA). However, use of the robotic technique is quite limited globally in part because of the high costs of the system and consumable equipment needed. Randomised controlled trials investigating chronic pain after thoracotomy versus VATS are rare, with studies sometimes limited to retrospective analysis of thoracotomy data, which is compared with prospectively collected data for VATS procedures (9).

Thoracic pain after surgery is partly nociceptive (somatic), related to the area of skin incision, and partly neuropathic, resulting from peripheral nerve (e.g., intercostal nerve) damage $(9,10)$. The former is part of the acute pain response, while the latter has been suggested as the major cause of post-thoracotomy pain syndrome (PTPS), that is, in the subacute or chronic phase. However, several studies have shown that PTPS is not only related to direct nerve injury (11-13). Acute postoperative thoracic pain is a strong predictor of PTPS (10), but the transition from acute to chronic pain is still poorly understood. Intense early postoperative pain is thought to cause neuroplastic changes, resulting in central sensitisation (14-16). Regional local anaesthetic (LA) techniques [thoracic epidural analgesia (TEA) or paravertebral blocks (PVBs)] are well established in the field of thoracic anaesthesia and particularly effective in limiting the development of chronification of pain. The use of LAs suppresses the nociceptive input in the acute postoperative period, which is believed to prevent central sensitisation (17).

\section{Why quantify intraoperative nociceptive levels?}

Currently, intraoperative administration of analgesia in thoracic surgery is mainly driven by either opioid analgesia or LA infusions, like thoracic epidural or PVBs. The former is delivered by population pharmacokinetic models of modern synthetic opioids incorporated into specific Target Controlled Infusion (TCI) devices to allow precise titration of blood and effect-site concentrations (18). Any further adjustments will depend on a patient's physiological responses such as sweating, increases in heart rate (HR) or blood pressure (BP), and the anaesthetist's clinical impression or experience. Inadequate opiate dosage intraoperatively may be associated with postoperative complications. For example, insufficient opiate use may lead to delayed recovery, prolonged hospital stays and chronic post-operative pain syndromes. Conversely, excessive opiate administration can result in nausea/vomiting, respiratory depression and hyperalgesia $(19,20)$. A more appropriate and patient-centred opioid exposure or even an opioid-free technique is now been suggested to minimise long-term opioid problems. This, however, is dependent on a more objective assessment of nociceptive stimulus leading to pain and inflammation. The success of LA infusions on the other side, depend on the accuracy in placing catheters and the effectiveness of dosing the LA drugs. Furthermore, the intensity of early postoperative pain correlates with the risk of developing a chronic pain state (21).

Monitoring quantitative measures of the 'nociceptionantinociception' balance may allow more personalised titration of opiate analgesia, thereby avoiding over- or under-dosing of opioids and other pain modulating agents, like ketamine or dexmedetomidine and their respective complications. There may also be an (as of yet unproven) relationship between intraoperative NAN levels and immediate post-operative pain.

\section{Physiology of nociception}

The nociceptive pathway of the body comprises the nociceptors, the ascending spinothalamic tracts, and the descending inhibitory tracts, as shown in Figure 1.

Nociceptors are free nerve endings, present in most body tissues and internal organs (22), which are activated by stimuli that cause or have the potential to cause tissue damage. Noxious stimuli include intense mechanical stimulation (e.g., skin incision), extremes of temperature (e.g., electrically-induced heat from a diathermy probe), and certain chemicals. There are 2 main nociceptor types: unmyelinated, slowly conducting $\mathrm{C}$ fibres, and thinly myelinated, faster conducting A $\delta$ fibres (23). The noxious 


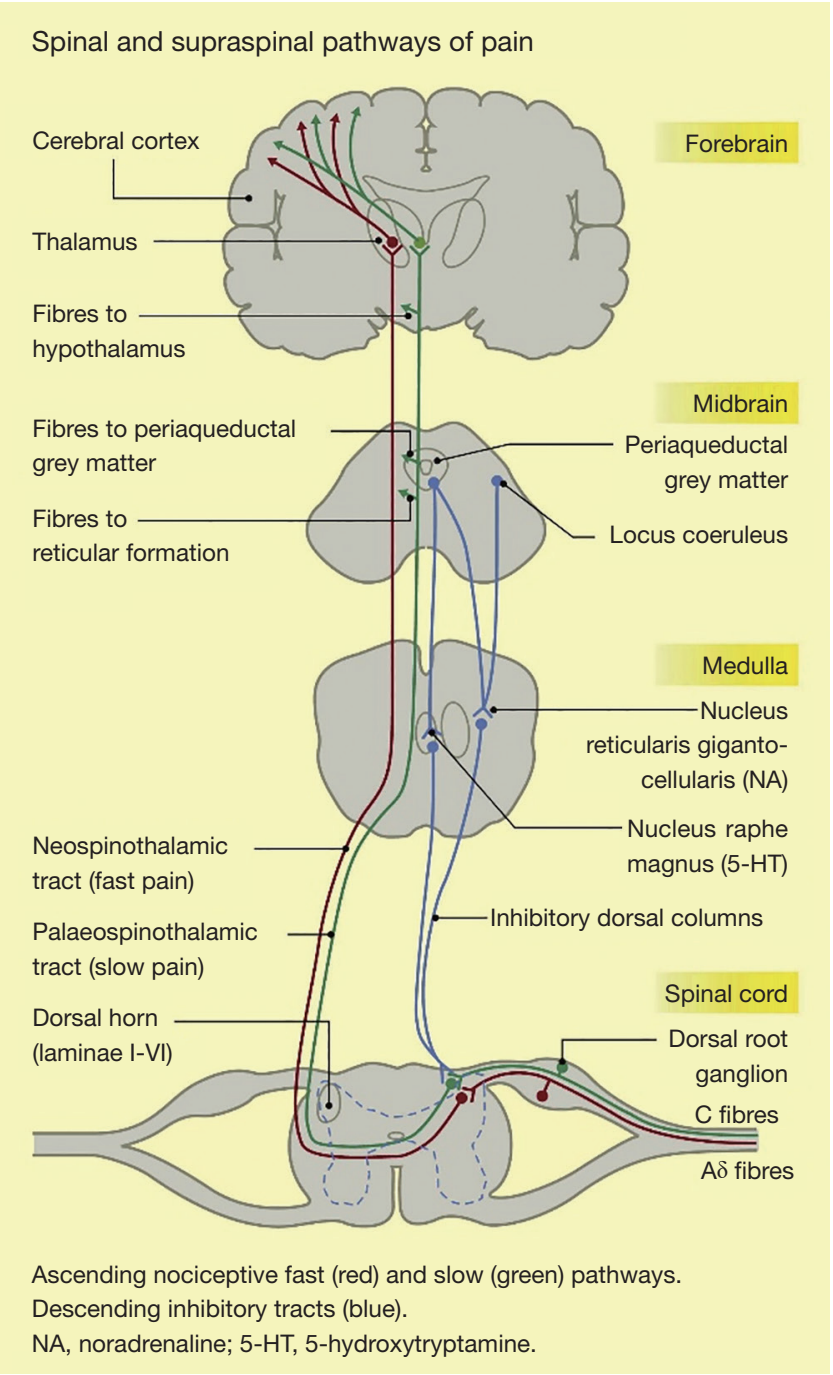

Figure 1 Ascending and descending nociceptive pathways in the body (Steeds, 2013) (15).

stimulus is transduced, i.e., converted into an electrical signal, which is conducted along these primary afferent or first-order neurons (24).

The first-order neurons have cell bodies in the dorsal root ganglia, and terminate in the dorsal horn of the spinal cord, where they give off ascending and/or descending branches, which rise or descend a short distance (one or two levels) within the zone of Lissauer, before synapsing with second-order neurons in the zona gelatinosa of the dorsal horn (22). These neurons decussate, i.e., cross over to the contralateral side of the spinal cord, and ascend in anterolateral spinothalamic tracts up to the thalamus. Here, they synapse with third-order neurons, which project on to the primary and secondary sensory cortices, as well as to other brain areas such as the prefrontal cortex, the amygdala, the insula, and the hypothalamus (24-26).

Descending pathways consists of neurons projecting from the sensory cortex to the hypothalamus and amygdala. The neurons synapse in the periaqueductal grey (PAG) in the midbrain. The PAG projects, via the rostral ventral medulla, down the spinal cord. The descending tracts can modulate the transmission of nociceptive information at the level of the dorsal horn $(25,26)$. Pain is therefore the combined result of a complex interplay between the nociceptive signalling pathways, other higher brain centres, and the emotional experience of a conscious individual. Given the variety of neurotransmitters and synapses involved in the nociceptive system of the body, there are multiple target sites for analgesics or antinociceptive agents to inhibit nociception or pain (26).

\section{Currently available nociceptive modalities}

Table 1 is an overview of some of the variables for monitoring the NAN balance, and the current evidence regarding their use.

\section{Analgesia Nociception Index (ANI)}

The ANI is measured using the CE-certified PhysioDoloris monitor (MetroDoloris Medical Systems, Lille, France), which uses electrocardiogram (ECG) signal output from a special patient sensor montage to construct an RR series $(27,28)$. The ANI is a surrogate measure of the relative parasympathetic activity of the autonomic nervous system, based on analysis of heart rate (RR interval) variability (HRV). HRV is controlled by the competing sympathetic and parasympathetic nervous system outflow to the sinoatrial node of the heart (29). There is evidence that high frequency fluctuations in $\mathrm{HRV}(>0.15 \mathrm{~Hz})$ are mediated by parasympathetic tone alone (29). The ANI is computed from the high-frequency component of HRV modulated by the effect of respiratory rate/rhythm (i.e., respiratory sinus arrhythmia) (27). More detailed descriptions of the methodology used are described elsewhere $(27,28)$. The output is a dimensionless value ranging from 0 to 100 . Increasing scores reflect increasing parasympathetic tone and decreasing nociceptive levels. A cut-off score of 50 has been suggested, with values $<50$ indicating inadequate nociception. The ANI performs an RR series analysis, with the RR samples isolated into windows of 


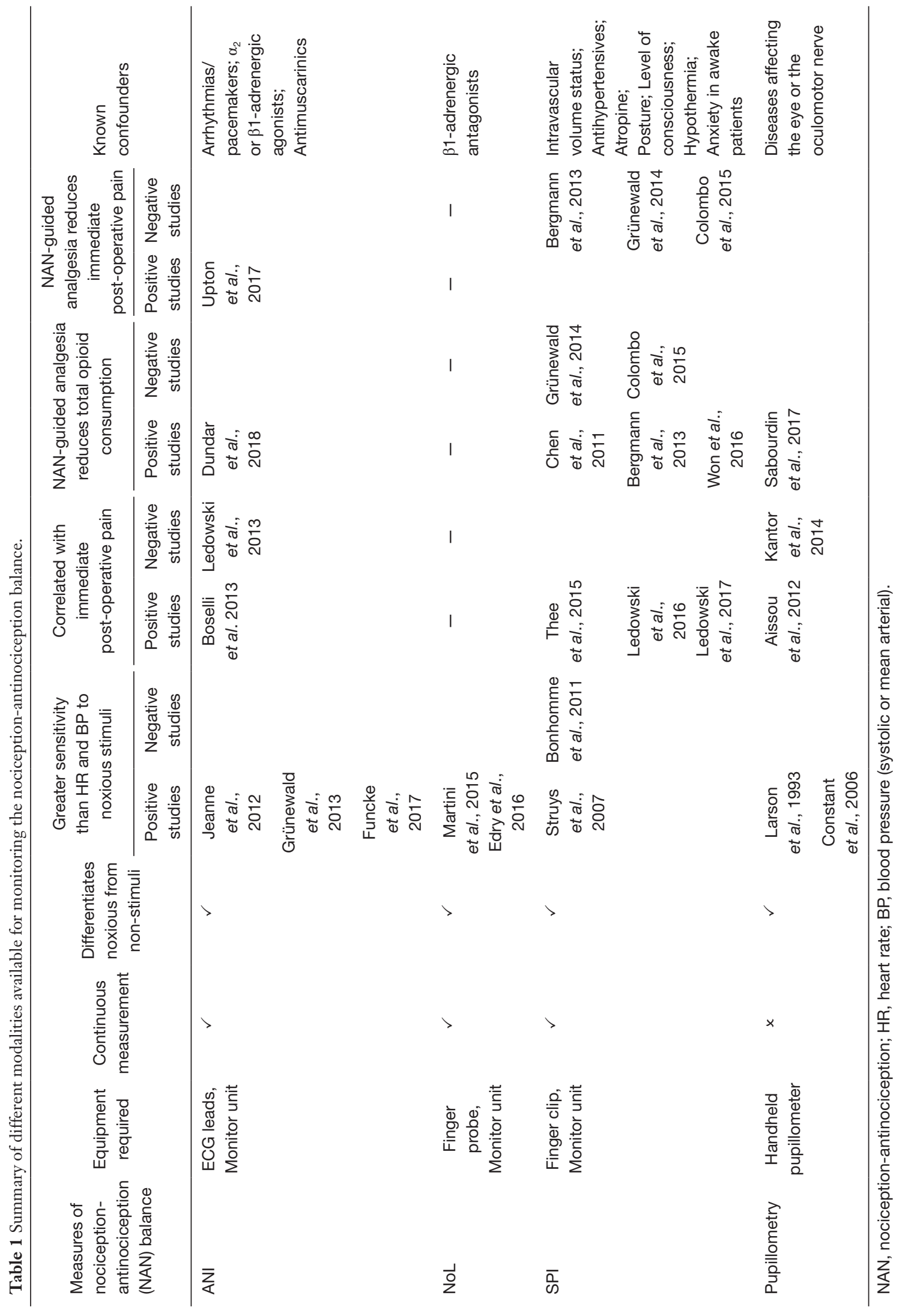


64 seconds to calculate the area under curve. Therefore, it could take 64 seconds for the first value to appear. It is recommended to allow for 3-4 time series to average and eliminate artefacts before a value is considered robust. This can indeed take some time but no longer that 4-5 minutes.

\section{Key study findings and limitations}

Jeanne et al. (30) reported a larger change in ANI compared to HR and systolic blood pressure (SBP), in response to noxious stimuli (pneumoperitoneum inflation) in propofolanaesthetised patients undergoing laparoscopic abdominal surgery. They concluded that the ANI is more sensitive than the standard haemodynamic responses of HR and BP to noxious stimuli. These findings were corroborated by Gruenewald and colleagues (31), who showed that the ANI value, but not HR or SBP, changed significantly after tetanic stimulation of the ulnar nerve in patients under propofol/ remifentanil anaesthesia. The ANI has also been shown to be superior to HR and mean arterial pressure (MAP) in detecting nociceptive tetanic stimulations under different doses of remifentanil (32).

However, correlating post-operative ANI levels with Numeric Rating Scale (NRS) pain scores has generated mixed results $(33,34)$. Of note, Ledowski et al. (33) showed that the ANI has poor sensitivity and specificity in differentiating between low (NRS 0) and high pain states (NRS 6-10) in the immediate post-operative period after sevoflurane anaesthesia.

The studies investigating use of intraoperative ANIguided anaesthesia in randomised controlled trials are possibly of greatest interest and clinical utility. For example, ANI-guided remifentanil anaesthesia has been observed to decrease intraoperative opioid consumption over standard infusion regulated according to haemodynamic parameters, in patients with thoracic paravertebral block (TPVB) undergoing breast surgery (35). In the 'ANI group', the remifentanil infusion rate was adjusted such that the ANI remained between 50 and 70 .

Finally, intraoperative ANI-guided fentanyl administration (to keep the ANI $\geq 50$ ) in patients undergoing lumbar discectomy and laminectomy decreased NRS scores in the immediate post-operative period ( $0-90$ minutes) by 1.3 units on average [ $95 \%$ confidence interval (CI): -0.4 to $2.4 ; \mathrm{P}=0.01]$ (36).

The main limitation of the ANI is that it cannot be used during arrhythmias, such as atrial fibrillation. ANI scores may also be unreliable in patients with implanted pacemakers, or those on drugs affecting autonomic tone, including $\alpha_{2}$-adrenergic agonists, $\beta_{1}$-adrenergic antagonists, and anti-muscarinics $(28,37)$.

\section{Nociception Level (NoL) Index}

The PMD-200 ${ }^{\mathrm{TM}}$ (Medasense Biometrics Ltd, Ramat Yishai, Israel) monitor, with a finger probe containing a plethysmography sensor and skin conductance electrodes are used to measure the NoL index (38). This index is derived from a non-linear combination of different physiological parameters, using Random Forest regression (38). The markers used are HR, the high-frequency component of HRV (i.e., at the $0.15 \mathrm{~Hz} 0.15$ - to $0.4-\mathrm{Hz}$ band power), photoplethysmography wave amplitude, skin conductance level, the number of skin conductance fluctuations, and their time derivatives. Ben-Israel et al. (38) who developed this method showed that the NoL increased significantly after minor to severe noxious stimuli. Furthermore, the NoL was superior to each of the individual variables from which it was derived, as well as their linear combination, in the assessment of the nociceptive response. The index ranges from 0 to 100 , with higher values indicating greater nociception. Medasense recommend a target NoL range of 10 to 25 .

\section{Key study findings and limitations}

Unlike the ANI, there have been a limited number of studies investigating the use of the NoL index, probably owing to the relative novelty of the technique. The first published study, investigating continuous measurement of HR, MAP, and the NoL index in 72 patients scheduled for elective surgery, showed that the NoL discriminated better noxious (skin incision and intubation) and nonnoxious events, compared to HR and MAP. The area under the receiver operating characteristic curve was greater for $\triangle \mathrm{NoL}(0.95)$ compared with $\triangle \mathrm{HR}(0.84, \mathrm{P}<0.001)$ or $\triangle \mathrm{MAP}$ $(0.78, \mathrm{P}<0.001)(39)$. Of note, a non-noxious stimulus was defined here as a 1 -minute interval within a 5 -minute period without any noxious stimulation. A subsequent validation study supported these findings, also showing that while the NoL increased progressively with increasing stimulus intensity, the response was blunted by administration of remifentanil at two different concentrations (40).

The effect of vasoactive drugs on the NoL index has not been elucidated yet. Studies have therefore excluded patients on $\beta$-adrenoreceptor blockers. Loss of signal strength for extended periods, while not mentioned in other studies, has been a common observation in our use 
of the NoL monitor, particularly during emergence from anaesthesia and in the awake patient.

\section{Surgical Pleth Index (SPI) [renamed from Surgical Stress Index (SSI)]}

Huiku and colleagues (41) created this index after investigating the effects of surgical stress on several physiological parameters in patients with varying opioid (remifentanil) concentrations. Parameters studied were the non-invasive systolic blood pressure (NIBP), normalised amplitude of the photoplethysmography wave (PPGA), pulse transit time (PTT), response entropy (RE), and normalised heart beat interval (HBI). Total surgical stress (TSS) for each patient was estimated using the surgical stimulus intensity and the remifentanil effect-site concentration $\left(\mathrm{Ce}_{\mathrm{remi}}\right)$. PPGA $\mathrm{Porm}_{\text {m }}$ correlated best with both the stimulus intensity the and the predicted $\mathrm{Ce}_{\text {remi }}$ level, while the PPT, NIBP and HBI ${ }_{\text {norm }}$ also showed decent correlation (41). As the NIBP is not usually continuously measured, and the PTT is prone to artifacts, the $\mathrm{HBI}_{\text {norm }}$ was selected along with the PPGA $\mathrm{Aorm}_{\text {no }}$ to create a twovariable model that would best predict the TSS. This model generated the surgical plethysmographic index (SPI), using the following formula:

SPI $=100-\left(0.7 \times \mathrm{PPGA}_{\text {norm }}+0.3 \times \mathrm{HBI}_{\text {norm }}\right)$

The SPI can be described as a measure of the sympathetic activity of the ANS (32).

The output is a dimensionless number ranging from 0 to 100 , with higher values indicating higher stress responses (41-43).

\section{Key study findings and limitations}

The SPI has been shown to discriminate noxious and nonnoxious stimuli in different surgical and anaesthetic settings $(41,44-48)$. However, it has not been shown to consistently outperform other standard haemodynamic variables in differentiating stimulus intensities. For example, in similarly designed studies, Struys et al. (48) and Bonhomme et al. (49) investigated the response of SPI and other haemodynamic variables (HR, RE, and $\mathrm{BP}$ ) to standard noxious stimuli under varying (remifentanil) concentrations. In the former, SPI correlated best with the opioid concentration; in the latter, there was no significant difference in the accurate prediction rate (APR) between the studied variables.

Several studies have investigated the relationship between intraoperative SPI levels and post-operative NRS pain scores in the PACU (post-anaesthesia care unit) in adults and children (50-52). In brief, the SPI has been shown to have moderate sensitivity and specificity in discriminating between low and moderate to severe pain, with for example, a SPI value $<40$ having a relatively high $(87.5 \%)$ negative predictive value (NPV) in ruling out significant postoperative pain in children (52).

There are now a number of published randomised controlled trials that have looked at the benefit of SPI-guided analgesia (maintaining an SPI $\leq 50$ ) over standard administration with adjustments made based on haemodynamic parameters (53-57). Again, there has been a lack of consistency in the results from these different studies. Some studies have reported reduced intraoperative opioid consumption $(53,54,57)$ and shorter recovery times [time to eye opening (54), extubation time (57)]. Others demonstrated similar opioid consumption, recovery times $(55,56)$, incidence of unwanted somatic events $(55)$, and NRS pain scores in the PACU (54-56).

Unfortunately, the SPI has several well-established limitations. It is influenced by any factors affecting sympathetic tone. Commonly mentioned confounders in the literature include intravascular volume status (57), cardiac pacemakers (58), atropine (59), antihypertensive drugs $(49,60)$, hypothermia (42), posture (61), level of consciousness or arousal $(51,62)$, and in awake patients, anxiety or emotional stress $(62,63)$.

\section{Pupillometry}

Pupil reflex Dilation (PRD) occurs in response to surgical nociceptive stimulation in anaesthetised patients, and pain, emotional stress or noxious stimulation in awake patients $(64,65)$. This reflex is thought to be mediated through reduction of parasympathetic tone in anaesthetised patients (66), and activation of the sympathetic nervous system in awake patients (65). There are several commercial portable infrared pupillometers available to measure pupil size such as the AlgiScan ${ }^{\circledR}$ (IDMed, Marseille, France, VideoAlgesiGraph ${ }^{\circledR}$ (Synapsys, Marseille, France), NeurOptics PLR-100 ${ }^{\circledR}$ (NeurOptics Inc., Irvine, Canada), and the Colvard pupillometer.

\section{Key study findings and limitations}

Larson et al. (67) reported a more significant increase in PRD compared to HR and SBP following electrical noxious stimulation in 13 volunteers under sevoflurane or propofol anaesthesia. Similar findings were reported by Constant and colleagues (68) following skin incision in 
24 children anaesthetised with sevoflurane. Other studies have demonstrated that the PRD correlates with the target plasma (69) or effect-site (70) concentration of opioid following tetanic stimulation in anaesthetised patients. Migeon et al. (71) investigated the use of PRD and the ANI, to assess the effectiveness of regional anaesthesia (RA) in children anaesthetised with sevoflurane. RA failure was defined as an increase in $\mathrm{HR} \geq 10 \%$ in the first 2 minutes after incision. Areas under the ROC curve for the maximal PRD and lowest ANI values were 0.671 and 0.741 respectively.

Guglielminotti and colleagues (72) showed that the PRD response to a standardised tetanic stimulation was as effective as (but not superior to) estimated remifentanil effect-site concentration in predicting movement following cervical dilatation. Therefore, the PRD could be an alternative in patients for whom the pharmacokinetic/ pharmacodynamic models used to derive the opioid Ce are not reliable, for example at the extremes of age or weight, and in shocked patients (72-74).

PRD in the immediate post-operative period was found to correlate significantly with pain intensity measured on a 5-point verbal rating scale (VRS) (75), but not with NRS (76) scores. In a single-group study, Huybrechts et al. (77) demonstrated that it is feasible to use PRD-guided thoracic epidural infusion (ropivacaine $0.5 \%$ ) in 13 patients undergoing thoracotomy under low-dose remifentanil/ propofol anaesthesia. In a more recent randomised study, pupillometry-guided intraoperative opioid administration decreased intraoperative remifentanil use compared to standard TCI practice in 55 women undergoing gynaecological procedures (78).

The main limitation of the PRD is that it cannot be continuously measured/monitored. Because it requires (brief) access to and exposure of the cornea, it is also not the most practical, with potential movement of the eyeball during anaesthesia making the measurement more difficult (43). There are also limitations in patients requiring prone positions. Lastly, it cannot be reliably measured in patients with certain diseases affecting the eye or the oculomotor nerve, and in those who have had previous eye surgery (73).

\section{How do these modalities perform against one another?}

There have been a small number of studies comparing the different approaches to nociception monitoring. Grünewald et al. (31) showed that both the ANI and SPI changed significantly after insertion of a laryngeal mask and tetanic stimulations under different effect-site concentrations of remifentanil. However, neither could significantly predict movement in response to these noxious stimuli, with prediction probabilities ( $\mathrm{P}$ values) being $0.41(0.13)$ for the ANI and $0.62(0.12)$ for the SPI. As mentioned earlier, in the study by Migeon and colleagues (71), the area under the ROC curve for identifying failure of RA was higher for the minimum value of the ANI (0.747) than the maximum value of the PRD (0.641). Measurements were taken during the first 2 minutes after skin incision. Finally, Stöckle et al. (79) demonstrated that the AUC for the response to tracheal intubation in 40 patients undergoing laparotomy was greater for the NoL than the ANI (0.93 vs. 0.84), but the opposite was true for the response to a standardised tetanic stimulus under different remifentanil infusion rates.

\section{Other drawbacks of NAN-monitoring research}

Apart from the specific limitations of each modality, there are more general issues regarding both the nociception indices and the design and conduct of research investigating their use. The response of an ideal NAN index should be independent of the depth of hypnosis. However, this is probably difficult to achieve both in theory and practice. It is not therefore clear how much of an effect propofol or other hypnotic agents have on each of the nociception markers.

Given the absence of a gold standard or even 'accepted' nociception monitor, validation of any new nociception modality is difficult (40). Using self-reported post-operative pain in the form of NRS scores is probably not the solution, as they have their own limitations too $(80,81)$. Much of the research so far has focused on relatively uniform patient populations (usually ASA grade I or II adults), undergoing a limited set of surgical procedures, or subject to standardised tetanic noxious stimuli. While limiting variability is important for statistical testing, it limits our ability to pick up potentially confounding factors.

A more reliable application of the NAN balance could be achieved by in studying the effectiveness of LA blocks as they reliably suppress both $\mathrm{A} \delta$ and $\mathrm{C}$ fibre conduction and therefore both somatic and visceral nociception as seen in thoracic anaesthesia and surgery. In the awake patient, (the extent of) sensory block can be easily tested by assessing the response to pinprick or ice at the targeted dermatomes. This is not possible in the anaesthetised, and hence, it is difficult 

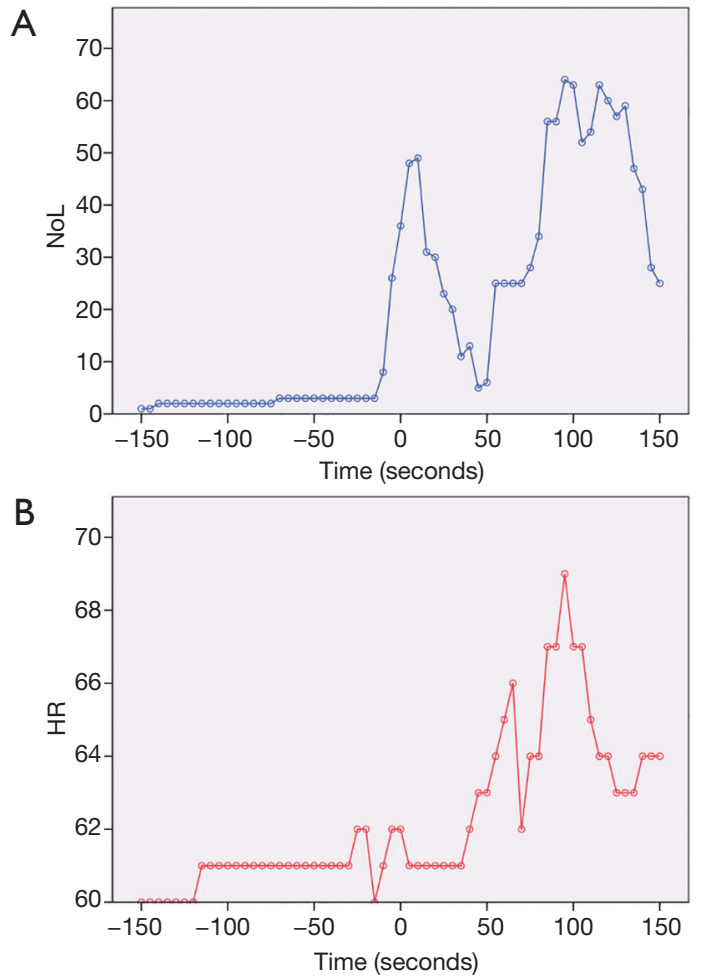

Figure 2 Variation of the NoL (A) and HR (B) in response to skin incision at time $=0 \mathrm{~s}$ and trocar insertion $15 \mathrm{~s}$ later.

to evaluate the success of LA techniques administered after induction of GA. Further research is needed to test the suitability of using NAN indices for assessing (or confirming) the sympatholytic effect of intraoperative LA techniques such as epidural or paravertebral analgesia in thoracic surgery.

\section{How does anti-nociception monitoring perform in thoracic surgery?}

The following figures illustrate the use of the PMD- $100^{\mathrm{TM}}$ monitor and its NoL index in standard thoracic procedures in our department.

All patients received a set of two paravertebral catheters that were primed with $20 \mathrm{~mL}$ of Levobupivacaine $0.5 \%$. PVB were topped up at the end of the procedure with $10-15 \mathrm{~mL}$ Levobupivacaine $0.25 \%$. Systemic intraoperative analgesia was multi-modal and consisted of low dose ketamine, dexamethasone, non-steroidals (ketorolac) and a target-controlled infusion of remifentanil to a moderate concentration of $3-3.5 \mathrm{ng} / \mathrm{mL}$.

Figure 2 shows a small portion of the recording of the
NoL and HR during a VATS (video-assisted thoracoscopic surgery) lobectomy. The PMD-100 ${ }^{\mathrm{TM}}$ monitor measures these variables at 5 -second intervals. The first skin incision was made at time $=0 \mathrm{~s}$, with a trocar inserted approximately 15 s later. The first peak in the NoL curve (Figure $2 A$ ) probably corresponds to the first skin incision, while the second might reflect a delayed response to the trocar insertion or further surgical stimulation. The general trends of the NoL and HR curves do appear to resemble each other. Nonetheless, the much larger change in the NoL compared to the HR (Figure $2 B$ ) exemplifies why NAN indices have been quite consistently shown to be more sensitive to noxious stimuli than HR or BP.

Figure $3 A$ shows the NoL index recorded at 5 -second intervals for the entire duration of a robotic-assisted thoracoscopic surgery (RATS) lobectomy. Broadly speaking, there are 3 major peaks, corresponding to the timings of intubation, first skin incision, and extubation. Medasense, the company manufacturing the PMD- $100^{\mathrm{TM}}$ monitor that measures the NoL index, suggest a target NoL range of 10 to 25 , with values below 10 indicating no nociceptive reaction, or excessive analgesia use. Looking at Figure $3 \mathrm{~A}$, apart from the 3 peaks mentioned earlier, there were only a few other occasions where the NoL rose above 25. This suggests that the patient's nociceptive response was reasonably well controlled throughout the operation. A contributing factor was likely a well working PVB inserted and topped-up at an early stage immediately after insertion of the video trocar. Conversely, the NoL was below 10 for quite extended periods. This indicates that there might have been scope to reduce the intraoperative opioid consumption (at least for this specific case). However, it remains to be seen how this would then influence post-operative pain or recovery.

Figure $3 B$ is a similar recording of the NoL for the entire length of a VATS lobectomy. The loss of the NoL signal, just before the 40-minute mark, for over 5 minutes, is of note here. This corresponded to the time of the first skin incision.

We have highlighted these examples to visualize the potential benefits of nociception monitoring in thoracic surgical patients to establish that neuraxial and other nerve blocks are working to the desired level as previous studies have failed to demonstrate and report consistently the effectiveness of both HTE and PVB $(82,83)$.

\section{Conclusions}

Despite the emergence of promising new technologies in 

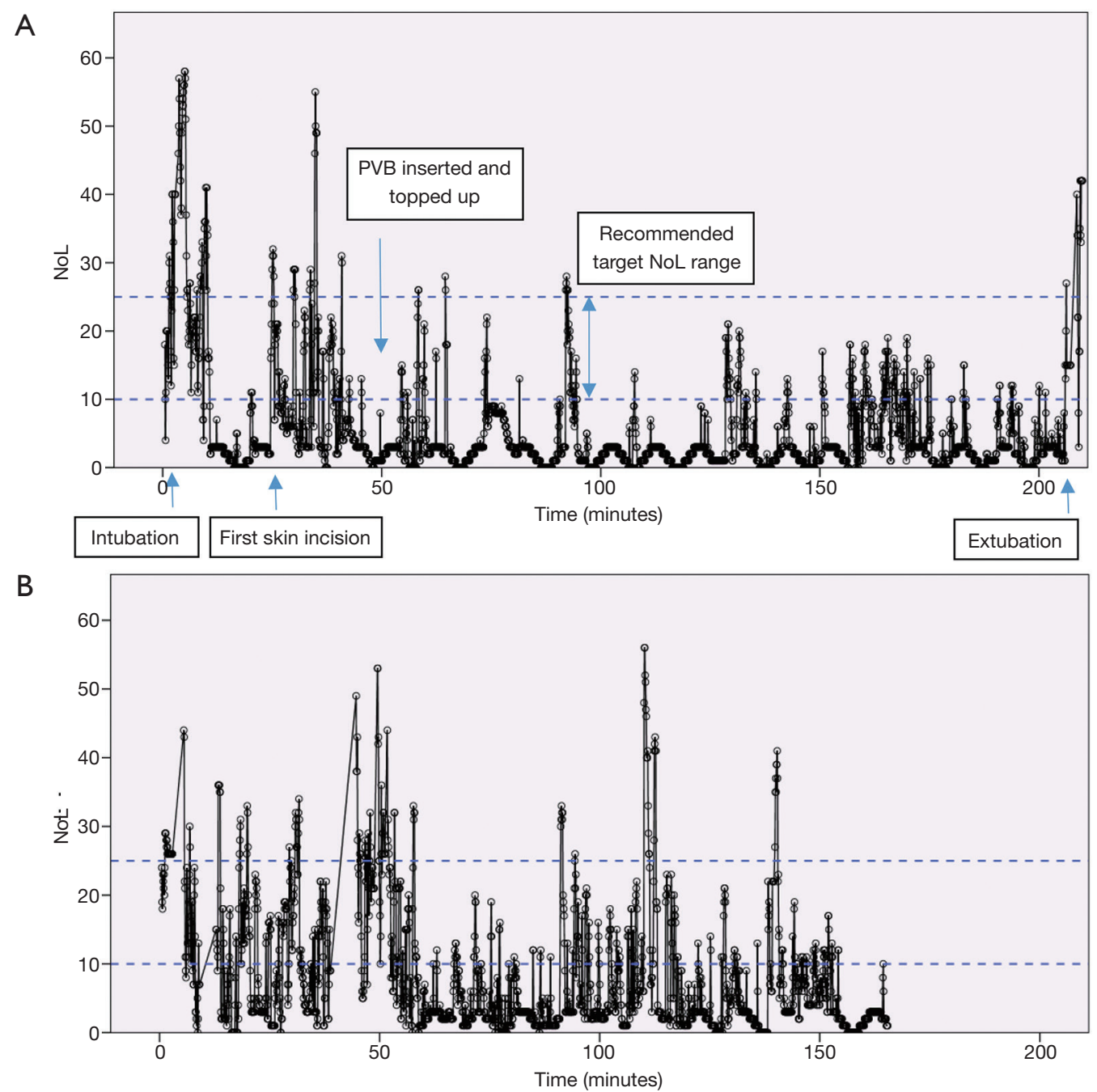

Figure 3 Course of the NoL during a RATS lobectomy case (A) and VATS lobectomy case (B).

recent years, detection and monitoring of the intraoperative NAN remains a challenge, and is not routinely carried out, other than for clinical research. In general, the different nociceptive measures available have been shown to be more sensitive to noxious stimuli than standard haemodynamic parameters. However, there is currently too little data to convincingly state that NAN indices can improve clinical practice and patient care. To properly evaluate the clinical utility of continuous nociceptive monitoring during surgery, larger randomised controlled trials are needed to investigate and then replicate the effect of NAN-guided anaesthesia on intraoperative opioid consumption, and post-operative outcomes compared to standard protocols. In particular, studying NAN-guided protocols during interventions that have a genuine physiological construct, such as thoracic epidural or para-vertebral LA blocks may be a starting point to gather more robust data. Ideally, this would involve different clinical settings, and wider, heterogeneous populations (including children and higher ASA grade patients).

Future anaesthetic strategies may eventually be heading towards simultaneous and independent monitoring of hypnosis and nociception, which may allow individualised titration of hypnotic and analgesic agents with the aim of controlling the nociception-anti-nociception balance intraoperatively, and pain post-operatively. The ultimate goal for thoracic surgeons and anaesthetists alike would be to reduce the incidence and severity of chronic pain after thoracic surgery, whether thoracotomy, VATs or RATs. 


\section{Acknowledgments}

None.

\section{Footnote}

Conflicts of Interest: The authors have no conflicts of interest to declare.

Ethical Statement: The authors are accountable for all aspects of the work in ensuring that questions related to the accuracy or integrity of any part of the work are appropriately investigated and resolved.

\section{References}

1. Brown EN, Lydic R, Schiff ND. General anesthesia, sleep, and coma. N Engl J Med 2010;363:2638-50.

2. Sigl JC, Chamoun NG. An introduction to bispectral analysis for the electroencephalogram. J Clin Monit 1994;10;392-404.

3. AL Vanluchene, H Vereecke, O Thas, et al. Spectral entropy as an electroencephalographic measure of anesthetic drug effect: a comparison with bispectral index and processed midlatency auditory evoked response. Anesthesiology 2004;101:34-42.

4. Lobo FA, Schraag S. Limitations of anaesthesia depth monitoring. Curr Opin Anaesthesiol 2011;24:657-64.

5. Hajat Z, Ahmad N, Andrzejowski J. The role and limitations of EEG-based depth of anaesthesia monitoring in theatres and intensive care. Anaesthesia. 2017;72 Suppl 1:38-47.

6. Cascella M. Mechanisms underlying brain monitoring during anesthesia: limitations, possible improvements, and perspectives. Korean J Anesthesiol 2016;69:113-20.

7. Loeser JD, Treede RD. The Kyoto protocol of IASP Basic Pain Terminology. Pain 2008;137:473-7.

8. Bayman EO, Brennan TJ. Incidence and severity of chronic pain at 3 and 6 months after thoracotomy: metaanalysis. J Pain 2014;15:887-97.

9. Nakazawa S, Shimizu K, Nagashima T, et al. Quality of life after video-assisted surgery for lung cancer. Lancet Oncol 2016;17:e318.

10. Blichfeldt-Eckhardt MR, Andersen C, Ørding H, et al. From acute to chronic pain after thoracic surgery: the significance of different components of the acute pain response. J Pain Res 2018;11:1541-8.

11. Wildgaard K, Ringsted TK, Aasvang EK, et al.
Neurophysiological characterization of persistent postthoracotomy pain. Clin J Pain 2012;28:136-42.

12. Steegers MA, Snik DM, Verhagen AF, et al. Only half of the chronic pain after thoracic surgery shows a neuropathic component. J Pain 2008;9:955-61.

13. Maguire MF, Latter JA, Mahajan R, et al. A study exploring the role of intercostal nerve damage in chronic pain after thoracic surgery. Eur J Cardiothorac Surg 2006;29:873-9.

14. Macrae WA. Chronic post-surgical pain: 10 years on. Br J Anaesth 2008;101:77-86.

15. Wildgaard K, Ringsted TK, Hansen HJ, et al. Quantitative sensory testing of persistent pain after video-assisted thoracic surgery lobectomy. Br J Anaesth 2012;108:126-33.

16. Katz J, Seltzer Z. Transition from acute to chronic postsurgical pain: risk factors and protective factors. Expert Rev Neurother 2009;9:723-44.

17. Crumley S, Schraag S. The role of local anaesthetic techniques in ERAS protocols for thoracic surgery. J Thorac Dis 2018;10:1998-2004.

18. Absalom AR, Mani V, De Smet T, et al. Pharmacokinetic models for propofol--defining and illuminating the devil in the detail. Br J Anaesth 2009;103:26-37.

19. Joly V, Richebe P, Guignard B, et al. Remifentanil-induced postoperative hyperalgesia and its prevention with smalldose ketamine. Anesthesiology 2005;103:147-55.

20. Fechner J, Ihmsen H, Schüttler J, et al. The impact of intra-operative sufentanil dosing on post-operative pain, hyperalgesia and morphine consumption after cardiac surgery. Eur J Pain 2013;17:562-70.

21. Gan TJ. Poorly controlled postoperative pain: prevalence, consequences, and prevention. J Pain Res 2017;10:2287-98.

22. Bear MF, Connors BW, Paradiso MA. Neuroscience: Exploring the brain. 4th Edition. Philadelphia: Wolters Kluwer, 2016.

23. Julius D, Basbaum AI. Molecular mechanisms of nociception. Nature 2001;413:203-10.

24. Boadas-Vaello P, Castany S, Homs J, et al. Neuroplasticity of ascending and descending pathways after somatosensory system injury: reviewing knowledge to identify neuropathic pain therapeutic targets. Spinal Cord 2016;54:330-40.

25. Steeds CE. The anatomy and physiology of pain. Surgery (Oxford) 2013;31:49-53.

26. Brown EN, Pavone KJ, Naranjo M. Multimodal General Anesthesia: Theory and Practice. Anesth Analg 2018;127:1246-58.

27. Jeanne M, Logier R, De Jonckheere J, et al. Validation of 
a graphic measurement of heart rate variability to assess analgesia/nociception balance during general anesthesia. Conference Proceedings: Annual International Conference of the IEEE Engineering in Medicine and Biology Society 2009;2009:1840-3.

28. Logier R, Jeanne M, De Jonckheere J, et al. PhysioDoloris: a monitoring device for analgesia/nociception balance evaluation using heart rate variability analysis. Conference Proceedings: Annual International Conference of the IEEE Engineering in Medicine and Biology Society 2010;2010:1194-7.

29. Task Force of the European Society of Cardiology the North American Society of Pacing Electrophysiology. Heart Rate Variability. Standards of Measurement, physiological interpretation and clinical use. Circulation 1996;93:1043-65.

30. Jeanne M, Clement C, De Jonckheere J, et al. Variations of the analgesia nociception index during general anaesthesia for laparoscopic abdominal surgery. J Clin Monit Comput 2012;26:289-94.

31. Gruenewald M, Ilies C, Herz J, et al. Influence of nociceptive stimulation on analgesia nociception index (ANI) during propofol-remifentanil anaesthesia. Br J Anaesth 2013;110:1024-30.

32. Funcke S, Sauerlaender S, Pinnschmidt HO, et al. Validation of Innovative Techniques for Monitoring Nociception during General Anesthesia. Anesthesiology 2017;127:272-83.

33. Ledowski T, Tiong WS, Lee C, et al. Analgesia nociception index: evaluation as a new parameter for acute postoperative pain. Br J Anaesth 2013;111:627-9.

34. Boselli E, Daniela-Ionescu M, Bégou G, et al. Prospective observational study of the non-invasive assessment of immediate postoperative pain using the analgesia/ nociception index (ANI) Br J Anaesth 2013;111:453-9.

35. Dundar N, Kus A, Gurkan Y, et al. Analgesia nociception index (ani) monitoring in patients with thoracic paravertebral block: a randomized controlled study. J Clin Monit Comput 2018;32:481-6.

36. Upton HD, Ludbrook GL, Wing A, et al. Intraoperative "Analgesia Nociception Index"- Guided Fentanyl Administration During Sevoflurane Anesthesia in Lumbar Discectomy and Laminectomy: A Randomized Clinical Trial. Anesth Analg 2017;125:81-90.

37. Silke B, Guy S, Riddell JG. Effects of beta-adrenoceptor agonists and antagonists on heart-rate variability in normal subjects assessed using summary statistics and nonlinear procedures. J Cardiovasc Pharmacol 1997;30:817-23.
38. Ben-Israel N, Kliger M, Zuckerman G, et al. Monitoring the nociception level: a multi-parameter approach. J Clin Monit Comput 2013;27:659-68.

39. Martini CH, Boon M, Broens SJ, et al. Ability of the Nociception Level, a Multiparameter Composite of Autonomic Signals, to Detect Noxious Stimuli during Propofol-Remifentanil Anesthesia. Anesthesiology 2015;123:524-34.

40. Edry R, Recea V, Dikust Y, et al. Preliminary Intraoperative Validation of the Nociception Level Index: A Noninvasive Nociception Monitor. Anesthesiology 2016;125:193-203.

41. Huiku M, Uutela K, van Gils M, et al. Assessment of surgical stress during general anaesthesia. Br J Anaesth 2007;98:447-55.

42. De Jonckheere J, Bonhomme V, Jeanne M, et al. Physiological Signal Processing for Individualized Antinociception Management During General Anesthesia: a Review. Yearb Med Inform 2015;10:95-101.

43. Gruenewald M, Ilies C. Monitoring the nociception-antinociception balance. Best Pract Res Clin Anaesthesiol 2013;27:235-47.

44. Ahonen J, Jokela R, Uutela K, et al. Surgical stress index reflects surgical stress in gynaecological laparoscopic daycase surgery. Br J Anaesth 2007;98:456-61.

45. Wennervirta J, Hynynen M, Koivusalo AM, et al. Surgical stress index as a measure of nociception/ antinociception balance during general anesthesia. Acta Anaesthesiol Scand 2008;52:1038-45.

46. Kallio H, Lindberg LI, Majander AS, et al. Measurement of surgical stress in anaesthetized children. Br J Anaesth 2008;101:383-9.

47. Gruenewald M, Meybohm P, Ilies C, et al. Influence of different remifentanil concentrations on the performance of the surgical stress index to detect a standardized painful stimulus during sevoflurane anaesthesia. Br J Anaesth 2009;103:586-93.

48. Struys MM, Vanpeteghem C, Huiku M, et al. Changes in a surgical stress index in response to standardized pain stimuli during propofol-remifentanil infusion. Br J Anaesth 2007;99:359-67.

49. Bonhomme V, Uutela K, Hans G, et al. Comparison of the surgical Pleth Index with haemodynamic variables to assess nociception-anti-nociception balance during general anaesthesia. Br J Anaesth 2011;106:101-11.

50. Thee C, Ilies C, Gruenewald M, et al. Reliability of the surgical Pleth index for assessment of postoperative pain: a pilot study. Eur J Anaesthesiol 2015;32:44-8. 
51. Ledowski T, Burke J, Hruby J. Surgical pleth index: prediction of postoperative pain and influence of arousal. Br J Anaesth 2016;117:371-4.

52. Ledowski T, Sommerfield D, Slevin L, et al. Surgical pleth index: prediction of postoperative pain in children? Br J Anaesth 2017;119:979-83.

53. Chen X, Thee C, Gruenewald M, et al. Comparison of surgical stress index-guided analgesia with standard clinical practice during routine general anesthesia: a pilot study. Anesthesiology 2010;112:1175-83.

54. Bergmann I, Gohner A, Crozier TA, et al. Surgical pleth index-guided remifentanil administration reduces remifentanil and propofol consumption and shortens recovery times in outpatient anaesthesia. Br J Anaesth 2013;110:622-8.

55. Gruenewald M, Willms S, Broch O, et al. Sufentanil administration guided by surgical pleth index vs standard practice during sevoflurane anaesthesia: a randomized controlled pilot study. Br J Anaesth 2014;112:898-905.

56. Colombo R, Raimondi F, Rech R, et al. Surgical Pleth Index guided analgesia blunts the intraoperative sympathetic response to laparoscopic cholecystectomy. Minerva Anestesiol 2015;81:837-45.

57. Won YJ, Lim BG, Lee SH, et al. Comparison of relative oxycodone consumption in surgical pleth index-guided analgesia versus conventional analgesia during sevoflurane anesthesia. Medicine (Baltimore) 2016;95:e4743.

58. Hans P, Verscheure S, Uutela K, et al. Effect of a fluid challenge on the Surgical Pleth Index during stable propofol-remifentanil anaesthesia. Acta Anaesthesiol Scand 2012;56:787-96.

59. Höcker J, Broch O, Gräsner JT, et al. Surgical stress index in response to pacemaker stimulation or atropine. Br J Anaesth 2010;105:150-4.

60. Won YJ, Lim BG, Yeo GE, et al. The effect of nicardipine on the surgical pleth index during thyroidectomy under general anesthesia: A prospective double-blind randomized controlled trial. Medicine (Baltimore) 2017;96:e6154.

61. Ilies C, Ludwigs J, Gruenewald M, et al. The effect of posture and anaesthetic technique on the surgical pleth index. Anaesthesia 2012;67:508-13.

62. Cowen R, Stasiowska MK, Laycock H, et al. Assessing pain objectively: the use of physiological markers. Anaesthesia 2015;70:828-47.

63. Ilies C, Gruenewald M, Ludwigs J, et al. Evaluation of the surgical stress index during spinal and general anaesthesia. Br J Anaesth 2010;105:533-7.

64. Chapman CR, Oka S, Bradshaw DH, et al. Phasic pupil dilation response to noxious stimulation in normal volunteers: relationship to brain evoked potentials and pain report. Psychophysiology 1999;36:44-52.

65. Yang LL, Niemann CU, Larson MD. Mechanism of pupillary reflex dilation in awake volunteers and in organ donors. Anesthesiology 2003;99:1281-6.

66. Larson MD, Tayefeh F, Sessler DI, et al. Sympathetic nervous system does not mediate reflex pupillary dilation during desflurane anesthesia. Anesthesiology 1996;85:748-54.

67. Larson MD, Sessler DI, Washington DE, et al. Pupillary response to noxious stimulation during isoflurane and propofol anesthesia. Anesth Analg 1993;76:1072-8.

68. Constant I, Nghe MC, Boudet L, et al. Reflex pupillary dilatation in response to skin incision and alfentanil in children anaesthetized with sevoflurane: a more sensitive measure of noxious stimulation than the commonly used variables. Br J Anaesth 2006;96:614-9.

69. Larson MD, Kurz A, Sessler DI, et al. Alfentanil blocks reflex pupillary dilation in response to noxious stimulation but does not diminish the light reflex. Anesthesiology 1997;87:849-55.

70. Barvais L, Engelman E, Eba JM, et al. Effect site concentrations of remifentanil and pupil response to noxious stimulation. Br J Anaesth 2003;91:347-52.

71. Migeon A, Desgranges FP, Chassard D, et al. Pupillary reflex dilatation and analgesia nociception index monitoring to assess the effectiveness of regional anesthesia in children anesthetised with sevoflurane. Paediatr Anaesth 2013;23:1160-5.

72. Guglielminotti J, Grillot N, Paule M, et al. Prediction of movement to surgical stimulation by the pupillary dilatation reflex amplitude evoked by a standardized noxious test. Anesthesiology 2015;122:985-93.

73. Larson MD, Gupta DK. Pupillary Reflex Dilation to Predict Movement: A Step Forward Toward Real-time Individualized Intravenous Anesthetics. Anesthesiology 2015;122:961-3.

74. Struys MM, Sahinovic M, Lichtenbelt BJ, et al. Optimizing intravenous drug administration by applying pharmacokinetic/pharmacodynamic concepts. Br J Anaesth 2011;107:38-47.

75. Aissou M, Snauwaert A, Dupuis C, et al. Objective assessment of the immediate postoperative analgesia using pupillary reflex measurement: a prospective and observational study. Anesthesiology 2012;116:1006-12 .

76. Kantor E, Montravers P, Longrois D, et al. Pain assessment in the postanaesthesia care unit using pupillometry: a 
cross-sectional study after standard anaesthetic care. Eur J Anaesthesiol 2014;31:91-7.

77. Huybrechts I, Barvais L, Ducart A, et al. Assessment of thoracic epidural analgesia during general anesthesia using pupillary reflex dilation: a preliminary study. J Cardiothorac Vasc Anesth 2006;20:664-7.

78. Sabourdin N, Barrois J, Louvet N, et al. Pupillometryguided Intraoperative Remifentanil Administration versus Standard Practice Influences Opioid Use: A Randomized Study. Anesthesiology 2017;127:284-92.

79. Stöckle PA, Julien M, Issa R, et al. Validation of the PMD100 and its NOL Index to detect nociception at different infusion regimen of remifentanil in patients under general anesthesia. Minerva Anestesiol 2018;84:1160-8.

Cite this article as: Ghanty I, Schraag S. The quantification and monitoring of intraoperative nociception levels in thoracic surgery: a review. J Thorac Dis 2019;11(9):4059-4071. doi: $10.21037 /$ jtd.2019.08.62
80. Krebs EE, Carey TS, Weinberger M. Accuracy of the pain numeric rating scale as a screening test in primary care. J Gen Intern Med 2007;22:1453-8.

81. Breivik H, Borchgrevink PC, Allen SM. Assessment of pain. Br J Anaesth 2008;101:17-24.

82. Joshi GP, Bonnet F, Shah R, et al. A systematic review of randomized trials evaluating regional techniques for postthoracotomy analgesia. Anesth Analg 2008;107:1026-40.

83. Yeung JH, Gates S, Naidu BV, et al. Paravetrebral block versus thoracic epidural for patients undergoing thoracotomy. Cochrane Database of Systematic Reviews 2016, issue 2. Art. No.: CD009121. 